\title{
Manejo de secuencia de Pierre Robin con distracción externa mandibular y palatoplastia de incisiones mínimas
}

\author{
Managment of Pierre Robin Sequence with external mandibular \\ distraction and palatoplasty of minimal incisions
}

José Israel Espino-Gaucín ${ }^{1}$, Carlos Eduardo Rodríguez Rodríguez', Carlos Altamirano Arcos', Luciano Nahas Combina ${ }^{1}$, Andrade Delgado Laura ${ }^{2}$, Héctor Malagon Hidalgo ${ }^{3}$

\section{RESUMEN}

Antecedentes. La secuencia de Pierre Robin es una patología poco frecuente entendida en la literatura latinoamericana . La incidencia en el mundo es variable y en Latinoamerica no existen cifras exactas. La etiopatogenia es poco clara y aminorar y manejar las complicaciones respiratorias constituyen un objetivo primordial en el tratamiento de estos pacientes. El objetivo de este artículo es presentar el manejo del Pierre Robin en nuestro Servicio desde un punto de vista íntegro presentando un caso ejemplo.

Presentación de caso. Masculino de 9 meses con diagnóstico de Pierre Robin, quien fue tratado con distractores mandibulares y retiro de los mismos tres meses después, con una excelente evolución posoperatoria. Posterior a esto, se realizó una palatoplastia sin complicaciones, con excelentes resultados respiratorios a los 6 meses de seguimiento.

Conclusiones. El manejo del síndrome de Pierre Robin es un desafío para el cirujano plástico, con muchas opciones quirúrgicas disponibles, pero la distracción mandibular es actualmente la técnica que tiene mejores resultados confirmados para los parámetros respiratorios a largo plazo.

\begin{abstract}
Background. Pierre Robin's sequence is a rare pathology. The incidence in the world is variable and in Latin America is unknown. The etiopathogenesis is unclear and respiratory complications are a challenge for surgical treatment. The objective of this article is to present the management of Pierre Robin from an integrative point of view through a case presentation.

Case presentation. 9 month old male with Pierre Robin diagnosis who was treated with mandibular distractors with subsequent removal 3 months later with excellent postoperative evolution. Later on, a la palatoplasty was performed with no complications, with good respiratory injuries function at 6 month follow up. Conclusions. The management of Pierre Robin syndrome is a challenge for the plastic surgeon, with many surgical options available, with mandíbular distraction currently being the technique of choice because of its confirmed favorable long term respiratory results.
\end{abstract}

Key words: XPierre Robin, glossoptosis, micrognathia, mandibular distractor.

Palabras claves: Pierre Robin, glosoptosis, micrognatia, distractor mandibular.

REVISTA ARGENTINA DE CIRUGÍA PLÁSTICA 2020;26(1):36-40. HTTPS:/DOI.ORG/10.32825/RACP/202001/0036-0040

\section{INTRODUCCIÓN}

La asociación entre micrognatia, paladar hendido y obstrucción de las vías respiratorias fue descrita por primera vez por St-Hilaire en $1822^{1}$. Pierre Robin acuñó el término glosoptosis para la obstrucción de la orofaringe por la lengua secundaria a la mandíbula hipoplásica y en 1934 se describió la tríada de glosoptosis, micrognatia y obstrucción de la vía aérea. La incidencia mundial se reporta en la literatura de 1 caso en

1. Residente de Cirugía Plástica y Reconstructiva. "Hospital General Manuel Gea González"

2. Jefe del Departamento de Cirugía Plástica y Reconstructiva. "Hospital General Manuel Gea González"

Profesor titular del Curso de Alta Especialidad en Cirugía Craneofacial avanzada en división de Estudios de Posgrado de la Facultad de Medicina UNAM. Centro Médico Issemym Toluca. México

\ Correspondencia: José Israel Espino Gaucin Cordles@hotmail. com, Israel.espino@gmail.com

Los autores no declaran conflictos de intereses

Recibido: 14/07/2019 / Aceptado: 24/10/2019
50.000 recién nacidos ${ }^{2,3}$. La mortalidad asociada es de 3,6 a $21 \%$ y es variable según los síndromes asociados. El paladar hendido es frecuente (73-90\%), y erróneamente se confunde con la tríada clásica, pero no es parte de la secuencia. Este se presenta como consecuencia de otras malformaciones, al presentar obstrucción mecánica en la fusión palatina y a los cambios en la musculatura de la cara y la bocá. El síndrome de Pierre Robin se asocia con otros síndromes como el síndrome de Stickler (34\%), el síndrome velocardiofacial (11\%) o se encuentra como una entidad única en el 20-50\% de los casos $^{6,7}$. El objetivo principal de este artículo es presentar el manejo actual de la secuencia de Pierre Robin en el Hospital General "Dr. Manuel Gea González", en México, sustentado con antecedentes y estudios, mostrando la efectividad del tratamiento a través de un caso ejemplo.

\section{CASO CLÍNICO}

Paciente masculino con secuencia de Pierre Robin desde su nacimiento. Antecedentes perinatales de sufrimiento fetal y parto por cesárea, al nacimiento con un 


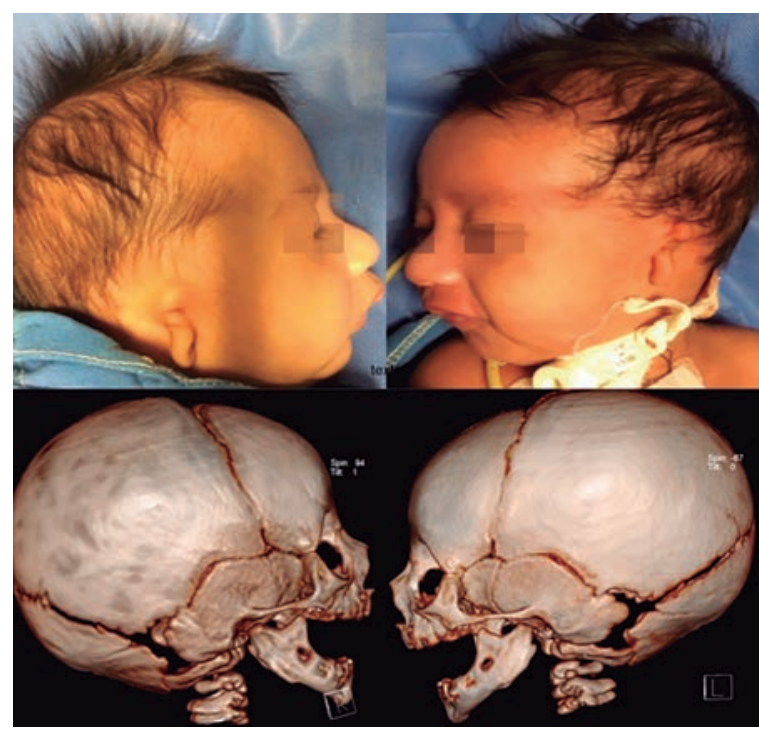

Figura 1.

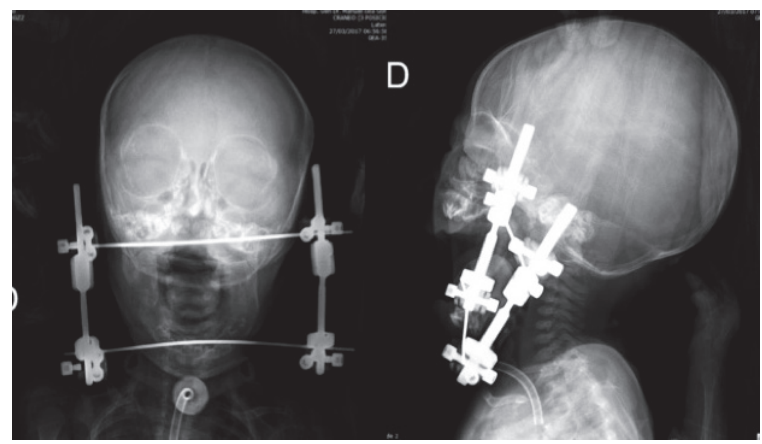

Figura 3.

peso de 2,3 kg y una altura de $45 \mathrm{~cm}$. Se programó al paciente para traqueostomía y gastrostomía al nacer debido a la obstrucción oral y de la vía aérea (Figura 1). La colocación de los distractores mandibulares se programó y desarrolló sin complicaciones a la edad de 2 meses, con un período posquirúrgico sin complicaciones y alta a su domicilio (Figura 2). La técnica utilizada consiste en hacer una incisión vestibular, disección del ángulo mandibular y una corticotomía externa oblicua del ángulo mandibular, que extiende la incisión hacia la cara superior de la mandíbula y hacia el borde inferior. Luego se coloca el distractor externo con tornillos laterales a la corticotomía y perpendicular a la mandíbula (Figura 3). La corticotomía oblicua realizada en el ángulo mandibular permite el crecimiento en vectores verticales y horizontales con $2 \mathrm{~mm}$ de distracción.

Una semana después se inició la distracción, avanzando $1 \mathrm{~mm}$ cada día hasta lograr los resultados requeridos, que en este caso fueron de 14-20 mm. Posteriormente, se inició un proceso de consolidación de 8 semanas, durante el cual se puede manipular el callo utilizando una mentonera para permitir una mejor oclusión.

El distractor se retira posterior a un control radiográfico en el que se vigila la formación de un callo óseo. En

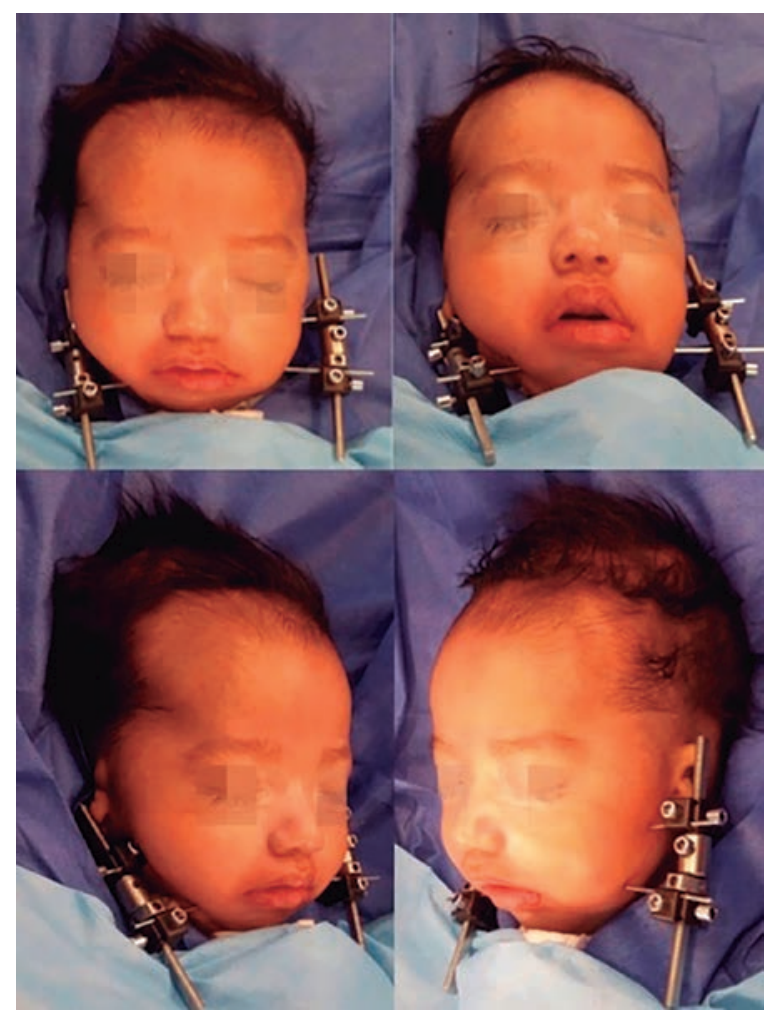

Figura 2.

este casodespués de tres meses de la colocación de distracción mandibular unidireccional, y secundario a los excelentes resultados, se retiró el dispositivo. Tres meses después de la cirugía, a la edad de 9 meses, se programó una palatoplastia con la técnica de incisiónes mínimas, la cual fue propuesta por el Dr. Mario Mendoza en nuestra unidad. Esta técnica consiste en una palatoplastia que se realiza con incisiones mediales en borde de la hendidura, con disección del mucoperiostio y liberación muscular, separando el plano de mucosa oral, realizando desincersión muscular la cual en la mayoria de los casos se encuentra en el borde de la hendidura, y mucosa nasal. Posteriormente se realiza cierre de mucosa nasal, reconstrucción de musculatura palatina, alineando y dando tensión a la musculatura del velo del paladar, y cierre de mucosa oral con sutura absorbible.

El paciente cursó un período posoperatorio satisfactorio y sin complicaciones (Figura 4). Actualmente, después de un año, el paciente sigue en control por nuestro Servicio de Cirugía Plástica, en donde se propone en un futuro realizarle una reconstrucción auricular bilateral, manejo de mordida abierta con servicios de Ortodoncia y Ortgnática y procedimientos secundarios, los cuales se completarán durante la niñez y la adolescencia (Figuras 5 y 6 ).

\section{DISCUSIÓN}

El gen exacto afectado o la etiopatogenia de Pierre Robin todavía se desconoce, aunque el gen $S O X 9$ se ha re- 


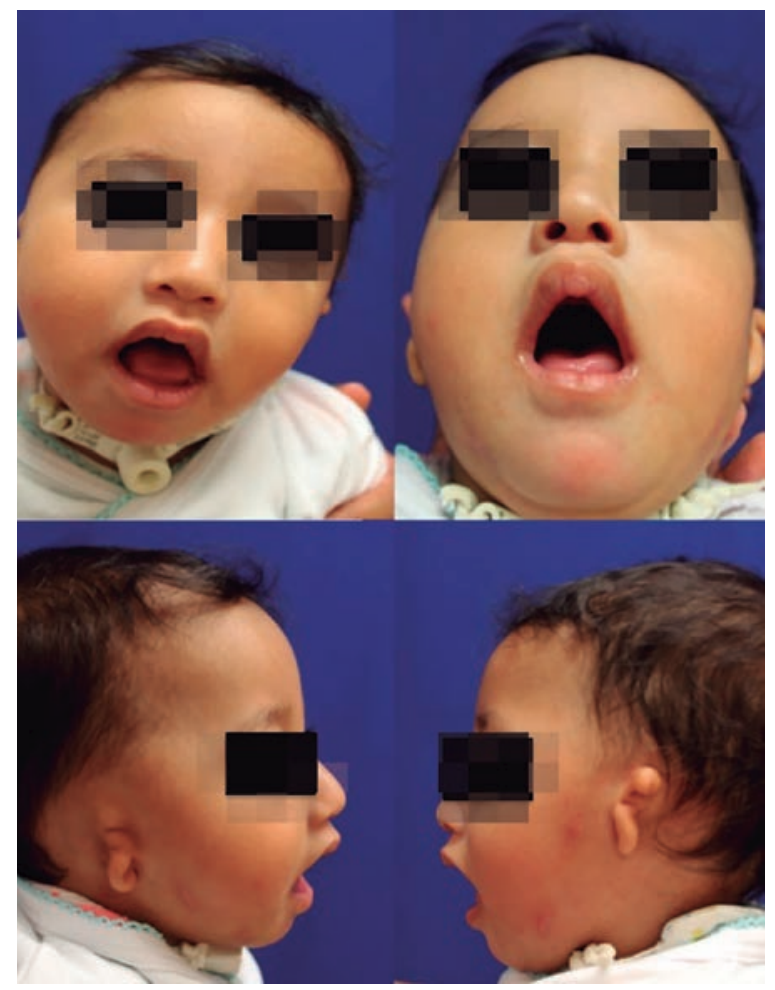

Figura 4.

lacionado, cuando no se presenta otro síndrome en familias con más de un miembro afectado ${ }^{8}$.

Existen diferentes teorías sobre la etiología del síndrome:

a. La teoría mecánica: el evento desencadenante es la hipoplasia de mandíbula.

b. La teoría de la maduración neurológica: secundaria a una neuroconducción alterada de músculos linguales y palatinos.

c. La teoría disneurológica rombencefálica: mala regulación en la organización motora del rombencefalo.

Las manifestaciones clínicas principales en esta secuencia son micrognatia y glosoptosis, que condicionarán la obstrucción de las vías respiratorias, así como otras alteraciones faciales clásicas. Por esta razón, los pacientes necesitan procedimientos quirúrgicos para garantizar la respiración y la alimentación correctamente, los cuales deben ser valorados desde el nacimiento y en los primeros meses de vida?.

La prevalencia de trastornos de la alimentación al nacimiento se estima en 25 a 45\%, los cuales están relacionados con problemas de ventilación y paladar hendido por lo que las recomendaciones para alimentar a estos pacientes es la posición en decúbito prono la cual minimiza la glosoptosis, pero esta posición no es aplicable para los recién nacidos. En estos casos los pacientes necesitarán tubos nasogástricos o gastrostomía ${ }^{11,12}$. El reflujo gastroesofágico también se ha identificado como un factor de riesgo para el fracaso de algunas maniobras terapéuticas ${ }^{13,14}$.

La obstrucción de la vía aérea es la principal alteración ventilatoria en los pacientes con Pierre Robin. El ron-

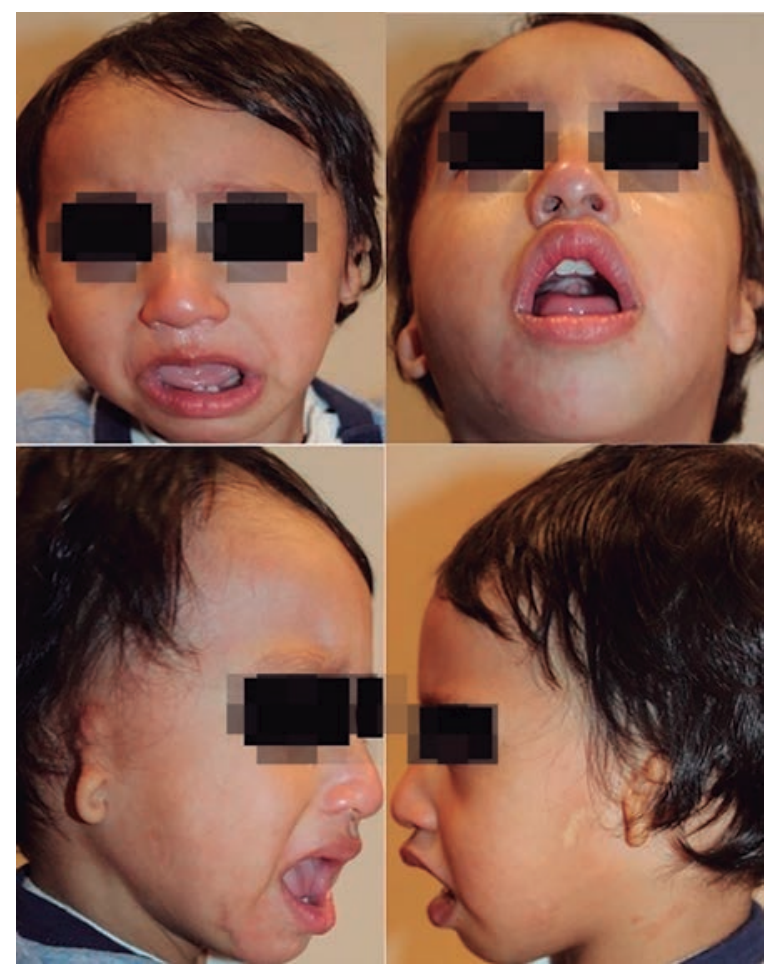

Figura 5.

quido es una manifestación cardinal de obstrucción de la vía aérea, pero no siempre está presente, y en algunas series solo el $50 \%$ presentan obstrucción grave asociada a ronquido, por lo que no es un signo fidedigno de obstrucción ${ }^{15,16}$.

Por otro lado, las anomalías de las vías respiratorias asociadas resaltan la necesidad de una evaluación cuidadosa mediante una nasolaringoscopia en todos los recién nacidos con secuencia de Pierre Robin, así como su referencia a un centro para atención multidisciplinaria por los servicios de Neonatología, Pediatria, Neumologia, Gastroenterología y Cirugía Plástica ${ }^{17,18}$. Por otro lado, el manejo quirúrgico se reserva para los casos en que el manejo conservador como la posición prona no son efectivos para despejar las vías respiratorias o el paciente no tolera la vía oral por compromiso respiratorio. La traqueotomía está reservada para pacientes con obstrucción severa de las vías respiratorias, los que presentan múltiples zonas de obstrucción, pacientes con menos de $2 \mathrm{~kg}$ que no son candidatos para la distracción mandibular o pacientes en quienes fracasó el tratamiento conservador. La sonda nasogástrica para alimentación o gastrostomía también puede ser necesaria, y debe ser una prioridad en la evaluación para mejorar peso y niveles nutricionales ${ }^{2,19}$.

Por otro lado, desde la introducción de la distracción mandibular, la glosopexia es menos usada, pero aun en nuestros días se describe como un tratamiento en los centros en donde no se tenga experiencia en cirugía craneofacial o en el uso de distractores mandibulares $^{19}$. Se reporta que se mantiene durante un año hasta que el paciente se encuentre en condiciones para rea- 


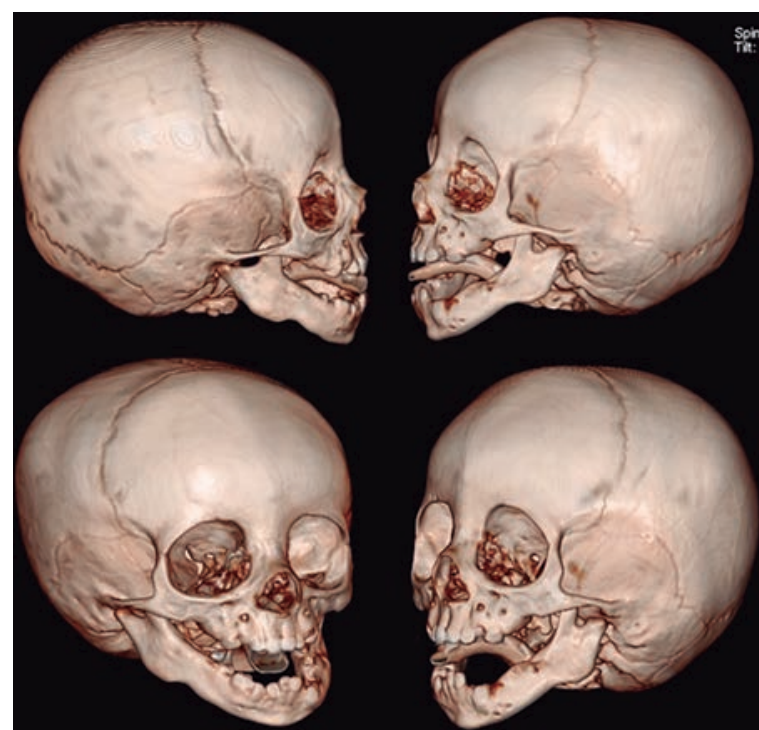

Figura 6.

lizar la palatoplastia, presentando un alivio inmediato de la obstrucción de la vía aérea ${ }^{20,21}$. La efectividad es de aproximadamente el $89 \%$, pero se reportan complicaciones en la alimentación y, a veces, el paciente necesita un tubo nasogástrico o gastrostomía para la alimentación. Entre las complicaciones se cuentan dehiscencias, abscesos, mucoceles y lesiones a conductos de Wharton ${ }^{22}$.

Por ultimo, la distracción mandibular ha sido una técnica utilizada ampliamente por nuestro servicio, que ha realizado importantes contribuciones por par- te del Dr. Fuente del Campo y el Dr. Fernando Molina a la literatura internacional ${ }^{23,24}$. La distracción mandibular se realiza para lograr un desplazamiento anterior de la lengua y aliviar la obstrucción de las vías respiratorias. Los pacientes toleran adecuadamente el uso de distractores, y las condiciones respiratorias y de alimentación mejoran gradualmente. Esto permite realizar el cierre del paladar a la edad de 9 meses, con la ventaja de una mejor respuesta fisiológica de la respiración y la deglución. Las complicaciones de este procedimiento incluyen la lesión de las ramas del nervio facial, del nervio alveolar inferior, lesiones de las raíces germinales dentales, cicatrices hipertróficas e infecciones ${ }^{25}$. En general, los pacientes sometidos a esta técnica logran una mejor saturación de oxígeno, con menor incidencia de apnea obstructiva del sueño y necesidad de traqueotomía que los pacientes sometidos a otras técnicas ${ }^{26}$.

\section{CONCLUSIONES}

El tratamiento de los pacientes con secuencia de Pierre Robin debe ser de tipo multidisciplinario y a largo plazo. Las manifestaciones clínicas incluyen la obstrucción de las vías respiratorias, trastornos de la alimentación y deglución. El manejo de estos pacientes es un reto para el cirujano plástico, ya que debe dar manejo a estructuras óseas y tejidos blandos, que permitirán mejorar la fisiología de las vías respiratorias, así como la armonía y la simetría facial.

\section{BIBLIOGRAFÍA}

1. Scott AR, Mader NS. Regional variations in the presentation and surgical management of Pierre Robin sequence: Regional Variations in PRS. The Laryngoscope. Diciembre de 2014;124(1):2818-25.

2. Cladis F, Kumar A, Grunwaldt L, Otteson T, Ford M, Losee JE. Pierre Robin Sequence: A Perioperative Review. AnesthAnalg. Agosto de 2014;119(2):400-12.

3. Robin P. Glossoptosis due to atresia and hypotrophy of the mandible. Arch Pediatr Adolesc Med. Septiembre de 1934:48(3):541.

4. Costa MA, Tu MM, Murage KP, Tholpady SS, Engle WA, Flores RL. Robin Sequence: Mortality, Causes of Death, and Clinical Outcomes. PlastReconstr Surg. Octubre de 2014;134(4):738-45.

5. Gangopadhyay N, Mendonca DA, Woo AS. Pierre Robin Sequence. Semin Plast Surg. Mayo de 2012;26(2):76-82.

6. Associated Conditions [Internet]. Pierre Robin Australia. Disponible en: http://www.pierrerobin.org.au/associated-conditions/

7. Côté A, Fanous A, Almajed A, Lacroix Y. Pierre Robin sequence: Review of diagnostic and treatment challenges. Int J PediatrOtorhinolaryngol. Abril de 2015;79(4):451-64.

8. Identification of Novel Craniofacial Regulatory Domains Located far Upstream of SOX9 and Disrupted in Pierre Robin Sequence - Gordon - 2014.

9. Buchanan EP, Xue AS, Hollier LH. Craniofacial Syndromes: PlastRe constr Surg. Julio de 2014;134(1):128e-153e.

10. Dr. Johannes Schubert, Dr. HeikoJahn, Dr. Markus Berginski. Experimental Aspects of the Pathogenesis of Robin Sequence. Cleft Palate Craniofac J. Julio de 2005:42(4):372-6.
11. Bütow K-W, Zwahlen RA, Morkel JA, Naidoo S. Pierre Robin sequence: Subdivision, data, theories, and treatment - Part 1: History, sub divisions, and data. Ann Maxillofac Surg. 2016;6(1):31-4.

12. Airway Management in Pierre Robin Sequence: The Vancouver Classi fication. - PubMed-NCBI [Internet]. Disponible en: https://www.ncbi. nlm.nih.gov/pubmed/?term=Airway+Management+in+Pierre+Robin+Sequence\%3A+The+Vancouver+Classification

13. RathéM, Rayyan M, Schoenaers J, Dormaar JT, Breuls M, VerdonckA, et al. Pierre Robin sequence: Management of respiratory and feeding complications during the first year of life in a tertiary referral centre. Int J PediatrOtorhinolaryngol. Agosto de 2015;79(8):1206-12.

14. Anderson ICW, Sedaghat AR, McGinley BM, Redett RJ, BosS EF, Ishman $S L$. Prevalence and severity of obstructive sleep apnea and snoring in infants with Pierre Robin sequence. Cleft Palate-Craniofacial J Off Publ Am Cleft Palate-Craniofacial Assoc. Septiembre de 2011;48(5):614-8.

15. Latepresentation of upper airway obstruction in Pierre Robin sequence / Archives of Disease in Childhood [Internet]. Disponible en: $h$ ttp:// adc.bmj.com/content/83/5/435

16. Fishman G, Zemel M, DeRowe A, Sadot E, Sivan Y, Koltai PJ. Fiber-optic sleep endoscopy in children with persistent obstructive sleep apnea: Inter-observer correlation and comparison with awake endoscopy. Int J Pediatr Otorhinolaryngol. Mayo de 2013;77(5):752-5.

17. Durr ML, Meyer AK, Kezirian EJ, Rosbe KW. Drug-Induced Sleep Endoscopy in Persistent Pediatric Sleep-Disordered Breathing After Adenotonsillectomy. Arch Otolaryngol Neck Surg. Julio de 2012;138(7):638. 
18. Glynn F, Fitzgerald D, Earley MJ, Rowley H. Pierre Robin sequence: an institutional experience in the multidisciplinary management of airway, feeding and serous otitis media challenges. Int J PediatrOtorhinolaryngol. Septiembre de 2011;75(9):1152-5.

19. Rogers GF, Murthy AS, A LR, Mulliken JB. The GILLS Score: Part I. Patient selection for tongue-lip adhesion in Robin Sequence. PlastReconstr Surg. 2011;128(1):243-251

20. Abel F, Bajaj Y, Wyatt M, Wallis $C$. The successful use of the nasopharyngeal airway in Pierre Robin sequence: an 11-year experience. Arch Dis Child. Abril de 2012;97(4):331-4.

21. Cohen S, Greathouse ST, Rabbani C, O'Neil J, Kardatzke M, Hall T, et al. Robin sequence: what the multidisciplinary approach can do. J MultidiscipHealthc. Marzo2017; Volume 10:121-32.

22. Kirschner RE, Low DW, Randall $P$, et al. Surgical airway management in Pierre Robin sequence: is there a role for tongue-lip adhesion? Cleft Palate Craniofac J. 2003;40(1):13- 18.
23. Fuente-del Campo A. Distracción Máxilo-Mandibular (generación ósea inducida). Cir Ciruj 1997; 65: 110-115

24. Molina F. Mandibular Distraction Osteogenesis: A Clinical Experience of the Last 17 Years. Jounal of Craniofacial Surgery. 2009;20(Suppl 2):1794-800.

25. Mingo KM, Sidman JD, et al. Use of External Distractors and the Role of Imaging Prior to Mandibular Distraction in Infants With Isolated Pierre Robin Sequence and Stickler Syndrome. JAMA Facial Plast Surg. 2016 Mar-Apr;18(2):95-100. doi: 10.1001/jamafacial.2015.1658

26. Zhang RS, Hoppe IC, Taylor JA, Bartlett SP. Surgical Management and Outcomes of Pierre Robin Sequence: A Comparison of Mandibular Distraction Osteogenesis and Tongue-Lip Adhesion. PlastReconstr Surg. 2018 Aug;142(2):480-509 